\title{
Emerging multidrug-resistant Candida duobushaemulonif infections in Panama hospitals: importance of laboratory surveillance and accurate identification
}

Ruben Ramos ${ }^{1 *}$, Diego H. Caceres ${ }^{2,3 *}$, Marilyn Perez ${ }^{1}$, Nicole Garcia ${ }^{1}$, Wendy Castillo ${ }^{1}$, Erika Santiago ${ }^{4}$, Jovanna Borace ${ }^{4}$, Shawn R. Lockhart ${ }^{2}$, Elizabeth L. Berkow ${ }^{2}$, Lizbeth Hayer ${ }^{5}$, Andres Espinosa-Bode ${ }^{2}$, Jose Moreno ${ }^{1}$, Brendan R. Jackson ${ }^{2}$, Jackeline Moran ${ }^{1}$, Tom Chiller ${ }^{2}$, Gloriela de Villarreal ${ }^{1}$, Nestor Sosa ${ }^{1}$, the Red Nacional de Vigilancia and Snigdha Vallabhaneni ${ }^{2}$ 1 Instituto Conmemorativo Gorgas de Estudios de la Salud, Panama City, Panama 2 Centers for Disease Control and Prevention, Atlanta, GA, United States of America. 3 Oak Ridge Institute for Science and Education (ORISE), Oak Ridge, Tennessee, United States of America, 4 Hospital Santo Tomas, Panama City, Panama. 5 Ministerio de Salud de Panama, Panama City, Panama.

\section{INTRODUCTION}

- Candida duobushaemulonii is a yeast that belongs to the $C$. haemulonii species complex along with $C$. haemulonii and $C$. haemulonii var. vulnera.

- It is closely related to $C$. auris, which is multidrug-resistant (MDR) and can cause outbreaks in the healthcare setting.

- Little is known about species in the $C$. haemulonii complex because infections are rare.

- C. haemulonii infections have involved skin wounds in patients with diabetes or peripheral vascular disease.

- C. duobushaemulonii has been documented to cause vulvovaginal candidiasis and chronic lower extremity wound infections.

- However, bloodstream infections with these organisms is thought to be rare.

\section{METHODS}

Given the emergence of $C$. auris, the Panamanian Ministry of Health implemented laboratory surveillance for C. auris in October 2016.

- Suspected C. auris isolates were forwarded to the Panamanian national reference laboratory.

- Suspected C. auris included isolates identified as Candida haemulonii, Candida famata, or Candida spp. by VITEK $2^{\circledR}$

- Definitive identification was made with MALDI-TOF and D1/D2 sequencing.

- Antifungal susceptibility testing (AFST) was conducted by VITEK $2^{\circledR}$ for all isolates, and four isolates underwent AFST by broth microdilution and Etest ${ }^{\oplus}$, which are the reference methods.

\section{RESULTS}

During November 2016-May 2017, the national microbiology reference laboratory at Instituto Conmemorativo Gorgas de Estudios de la Salud (ICGES), Panama City, Panama, received 36 isolates suspected to be $C$. auris from 31 patients (All were initially identified as C. haemulonii).

Figure 1. Flow chart of suspected $C$. auris isolates

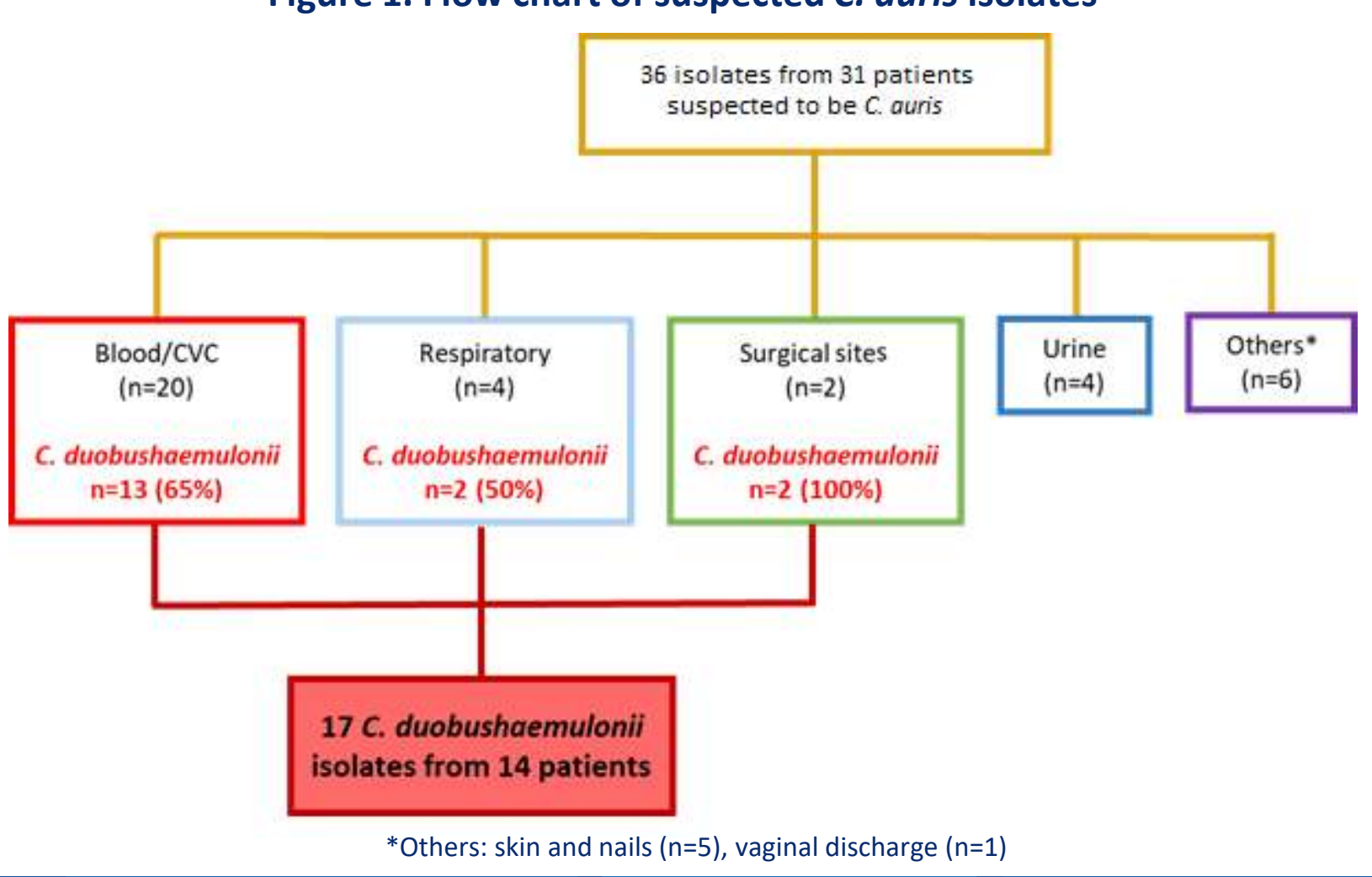

Patients

14 patients with $C$. duosbushaemulonii infection:

- Nine (64\%) patients were male.

- Mean age was 47 years (range: 0-77); three cases among children (two were 2 years old and one was $<30$ days old).

- Patients were treated in six hospitals from four geographical regions of Panama (Figure 1A); half were from one hospital in Panama City.

- More than half of patients were identified in the last two months of 2016 (Figure 1B)

Figure 1. Confirmed isolates of the merging pathogen C. duobushaemulonii in Panama, November 2016-May 2017.
ing
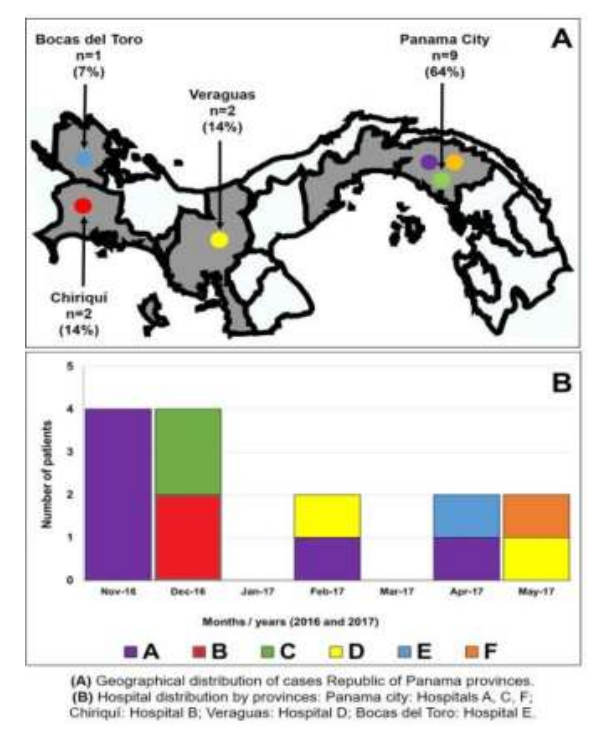

AFST results by VITEK $2^{\circledR}$

\begin{tabular}{|c|c|c|c|c|c|}
\hline \multicolumn{6}{|c|}{ Panama, 2016-2017 ( $n=17)$} \\
\hline & FCZ & VCZ & CSF & MCF & AmpB \\
\hline $\mathrm{MIC}_{50}$ & $32 \mu \mathrm{g} / \mathrm{mL}$ & $1 \mu \mathrm{g} / \mathrm{mL}$ & $0.25 \mu \mathrm{g} / \mathrm{mL}$ & $0.125 \mu \mathrm{g} / \mathrm{mL}$ & $>16 \mu \mathrm{g} / \mathrm{ml}$ \\
\hline $\mathrm{MIC}_{90}$ & $>64 \mu \mathrm{g} / \mathrm{mL}$ & $>8 \mu \mathrm{g} / \mathrm{mL}$ & $0.25 \mu \mathrm{g} / \mathrm{mL}$ & $0.25 \mu \mathrm{g} / \mathrm{mL}$ & $>16 \mu \mathrm{g} / \mathrm{ml}$ \\
\hline & & & 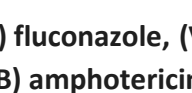 & & \\
\hline
\end{tabular}

AFST test by VITEK $2^{\circledR}$ revealed elevated MICs for fluconazole $(\geq 32 \mu \mathrm{g} / \mathrm{mL} ; \mathrm{n}=11 ; 65 \%)$ voriconazole ( $\geq 4 \mu \mathrm{g} / \mathrm{mL} ; \mathrm{n}=8 ; 47 \%$ ) and amphotericin B ( $\geq 2 \mu \mathrm{g} / \mathrm{mL} ; \mathrm{n}=14 ; 82 \%)$.

AFST conducted at CDC for four isolates using broth microdilution and $\mathrm{E}$ test:

- All had MICs for fluconazole of $\geq 32 \mu \mathrm{g} / \mathrm{mL}$ by VITEK $2^{\circledR}$, two had fluconazole MICs below the breakpoint ( 4 and $8 \mu \mathrm{g} / \mathrm{mL}$ ) by broth microdilution.

- Results for amphotericin B showed consistently high MICs, all $>8 \mu \mathrm{g} / \mathrm{mL}$ by both VITEK $2^{\circledR}$ and Etest ${ }^{\circledR}$.

- Although individual MIC values for micafungin varied slightly between AFST methodologies, the interpretation of susceptible remained regardless of method used.

Limitations

- We did not have clinical information on the patients, including, clinical conditions, treatments, and outcomes.

- We were unable to perform AFST by the reference methodology in 13 of 17 .

\section{CONCLUSIONS}

- We documented the presence of invasive infections by $C$. duobushaemulonii, a multidrug-resistant Candida species in Panama.

- Most C. duobushaemulonii isolates were cultured from the bloodstream establishing it as a cause of invasive infections. In fact, a majority of bloodstream isolates suspected of being $C$. auris were actually $C$. duobushaemulonii.

- Clinicians, microbiologists, and laboratory technicians should pay special attention to uncommon or unidentified fungal species identified by automated tests. Confirmatory testing with MALDI-TOF or DNA sequencing is recommended for these pathogens.

- Integrated epidemiologic and laboratory surveillance is a fundamental component of global health security and necessary for the detection and control of such emerging pathogens.

Mycotic Diseases Branch. Centers for Disease Control and Prevention (CDC) xju7@cdc.gov 